\title{
Reference Value for Infrapatellar Branch of Saphenous Nerve Conduction Study: Cadaveric and Clinical Study
}

\author{
Keon-tae Kim, MD, Yong-ki Kim, MD, Jung Ro Yoon, MD, Yundam Ko, MD, Myung Eun Chung, MD, PhD
}

Department of Rehabilitation Medicine, St. Paul's Hospital, College of Medicine, The Catholic University of Korea, Seoul, Korea

Objective To determine the optimal stimulation and recording site for infrapatellar branch of saphenous nerve (IPBSN) conduction studies by a cadaveric study, and to confirm that obtained location is practically applicable to healthy adults.

Methods Twelve lower limbs from six cadavers were studied. We defined the optimal stimulation site as the point IPBSN exits the sartorius muscle and the distance or ratio were measured on the X-and Y-axis based on the line connecting the medial and lateral poles of the patella. We defined the optimal recording site as the point where the terminal branch met the line connecting inferior pole of patella and tibial tuberosity, and measured the distance from the inferior pole. Also, nerve conduction studies were performed with obtained location in healthy adults.

Results In optimal stimulation site, the mean value of X-coordinate was $55.50 \pm 6.10 \mathrm{~mm}$, and the ratio of the Y-coordinate to the thigh length was $25.53 \% \pm 5.40 \%$. The optimal recording site was located $15.92 \pm 1.83 \mathrm{~mm}$ below the inferior pole of patella. In our sensory nerve conduction studies through this location, mean peak latency was $4.11 \pm 0.30 \mathrm{~ms}$ and mean amplitude was $4.16 \pm 1.49 \mu \mathrm{V}$.

Conclusion The optimal stimulation site was located 5.0-6.0 cm medial to medial pole of the patella and $25 \%$ of thigh length proximal to the X-axis. The optimal recording site was located $1.5-2.0 \mathrm{~cm}$ below inferior pole of patella. We have also confirmed that this location is clinically applicable.

Keywords Electromyography, Knee injuries, Cadaver

\section{INTRODUCTION}

The infrapatellar branch of saphenous nerve (IPBSN) is a purely sensory branch of the femoral nerve, originat- ing from the third and fourth lumbar nerve roots. Within the proximal third of thigh, the nerve enters the adductor canal. Here, the nerve joins with descending genicular artery and both the nerve and the artery pierce the ad-

Received May 24, 2017; Accepted July 19, 2017

Corresponding author: Myung Eun Chung

Department of Rehabilitation Medicine, St. Paul's Hospital, College of Medicine, The Catholic University of Korea, 180 Wangsan-ro, Dongdaemun-gu, Seoul 02559, Korea. Tel: +82-2-958-2307, Fax: +82-2-968-2307, E-mail: coltrane@catholic.ac.kr

ORCID: Keon-tae Kim (http://orcid.org/0000-0001-9174-909X); Yong-ki Kim (http://orcid.org/0000-0002-2116-0500); Jung Ro Yoon (http://orcid. org/0000-0002-3323-3738); Yundam Ko (http://orcid.org/0000-0003-0439-8063); Myung Eun Chung (http://orcid.org/0000-0002-7308-2815).

(c) This is an open-access article distributed under the terms of the Creative Commons Attribution Non-Commercial License (http://creativecommons.org/ licenses/by-nc/4.0) which permits unrestricted noncommercial use, distribution, and reproduction in any medium, provided the original work is properly cited. Copyright ( 2018 by Korean Academy of Rehabilitation Medicine 
ductor canal. After the saphenous nerve exits the adductor canal, it gives rise to the IPBSN. The IPBSN runs under the sartorius muscle. After penetrating the sartorius muscle and fascia lata to become subcutaneous, it runs superficially over the anteroinferior and medial aspect of knee [1-5]. IPBSN is connected to the medial and intermediate femoral cutaneous nerve and lateral femoral cutaneous nerve in the proximal to the knee, and to the other branches of the saphenous nerve in the distal to the knee, to form a cutaneous network called the peripatellar plexus [6].

IPBSN injury may result from non-traumatic, traumatic, or iatrogenic cause. Especially, there are numerous reports on IPBSN injury due to various surgical procedures on the medial aspect of knee [7-9].

Since patients with IPBSN injury suffer solely reported sensory abnormality around the knee (a subjective complaint dependent upon patient report) and in the absence of objective evidence such motor weakness, many clinicians overlook other evidence of trauma during clinical evaluation, diagnosis. This, in turn, effects the nature and duration of the treatment plan. Furthermore, the source of the knee pain is not always clear; knee/leg pain may be mistaken for an abnormality arising from a variety of anatomic structures, focal to the knee joint or actually emanating from another body part, such as the lumbar spine in the form of radicular symptoms or actual lumbar radiculopathy $[10,11]$. Therefore, IPBSN nerve conduction studies are critical and integral for the purpose of ruling in, or gruling out, a focal knee joint problem or medical issue arising from other etiology when IPBSN injury is suspected.

However, there have been documented variations in course and branching pattern of IPBSN by several studies and in particular, anatomical reference point(s) for IPBSN nerve conduction studies have not, as yet, been established. The aim of this study was to investigate the relative location of the IPBSN based on anatomical landmarks through cadaveric study, and to identify the optimal stimulation and recording point for use in IPBSN nerve conduction studies. In addition, we performed sensory nerve conduction studies at the obtained optimal stimulation and recording site to confirm that the obtained location is clinically applicable, and to propose the reference value.

\section{MATERIALS AND METHODS}

\section{Anatomical study}

Twelve lower limbs harvested from six fresh cadav-
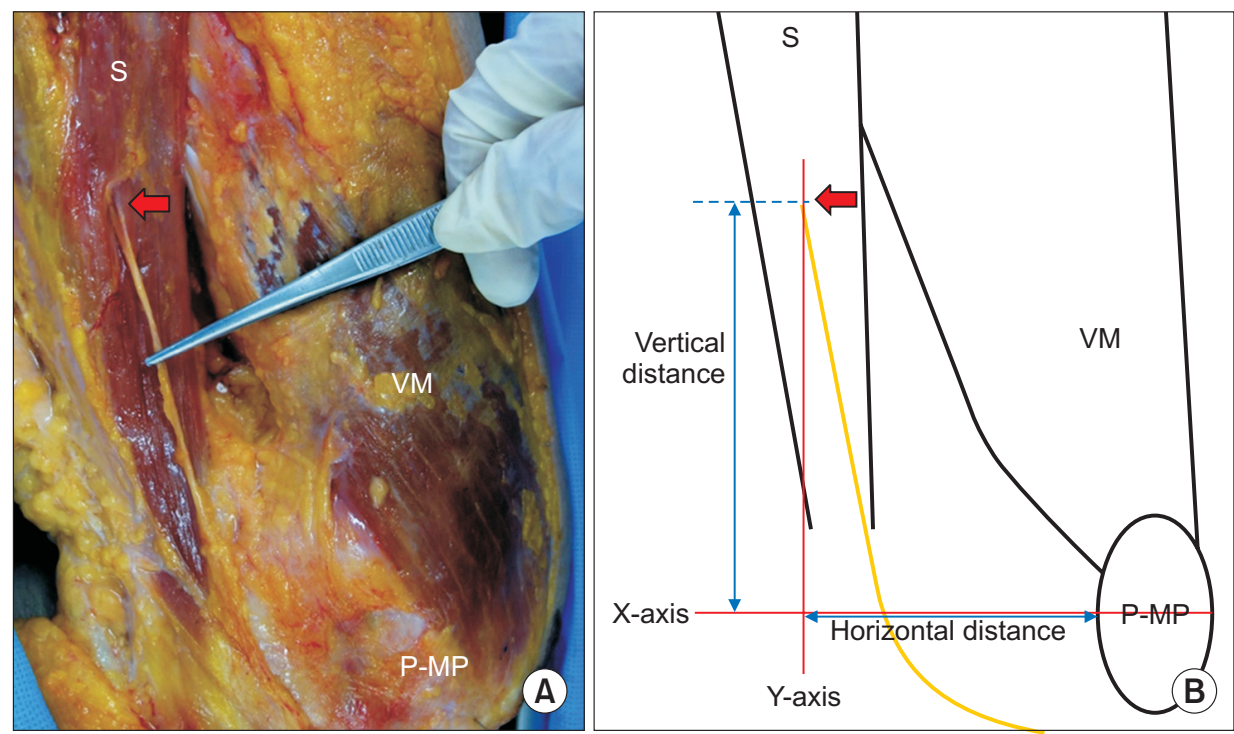

Fig. 1. (A) Anteromedial view of a dissected left knee showing the optimal stimulation site, where the IPBSN exit the sartorius muscle (arrow) in thigh. (B) Schematic illustration demonstrating anatomical landmarks and coordinates of stimulation site of IPBSN (arrow). The horizontal distance was from the medial pole of patella to the point where $\mathrm{X}$ - and $\mathrm{Y}$-axis cross. The vertical distance was from the point where the nerve exiting the sartorius muscle to the point where X- and Y-axis cross. VM, vastus medialis muscle; S, sartorius muscle; P-MP, medial pole of patella; IPBSN, infrapatellar branch of saphenous nerve. 
ers (two males and four females; median age, 69 years; range, 55-80 years) were used in this study. Specimens with deformities-such as contractures-or sequelae arising from a history of trauma or surgery of the knees were excluded. All the specimens were in supine position with their legs extended. The skin and subcutaneous tissues of the anterior and medial side from proximal thigh to the proximal shaft of tibia were carefully debrided and excised to lay bare the course of the IPBSN around the sartorius muscle and to identify the terminal branching pattern in front of the patellar ligament of the IPBSN.

The optimal stimulation site was defined as the location where the IPBSN exits the sartorius muscle to subcutaneous layer, since stimulation at the superficial location of the nerve can avoid volume conduction of motor potentials with minimal effective currents. The medial and lateral poles of the patella were designated 'bony landmarks' for reference purposes. The horizontal line connecting the medial and lateral poles of patella were defined as the 'reference line for X-coordinate (X-axis)'. The vertical line, perpendicular to the $\mathrm{X}$-axis, connecting the point where the IPBSN exits the sartorius muscle, was defined as the 'reference line for Y-coordinate (Y- axis)' The X-coordinate was the distance from the medial pole of patella to the point where $\mathrm{X}$ - and $\mathrm{Y}$-axis cross. The Y-coordinate was the distance from the point where the nerve exiting the sartorius muscle to the point where $\mathrm{X}$ - and Y-axis cross (Fig. 1). To compensate for the difference of the thigh length of individual, the Y-coordinate to thigh length ratio was determined in percentage. The distance from the mid-point between anterior superior iliac spine (ASIS) and pubic tubercle to the medial pole of patella was defined as thigh length.

We defined the optimal recording site as the 'location where the IPBSN terminates in front of or below the patella', because of least variation in the course of the IPBSN considering different thigh lengths. The inferior pole of patella and tibial tuberosity was used as reference bony landmarks. The number and location of terminal branch of IPBSN were also observed. If there were more than two branches, the most superior branch was selected for measuring, because it passes or terminates below the inferior pole of patella. The vertical line connecting inferior pole of patella and tibial tuberosity was defined as the 'reference line for optimal recording site.' The distance from the inferior pole of the patella to the point where the
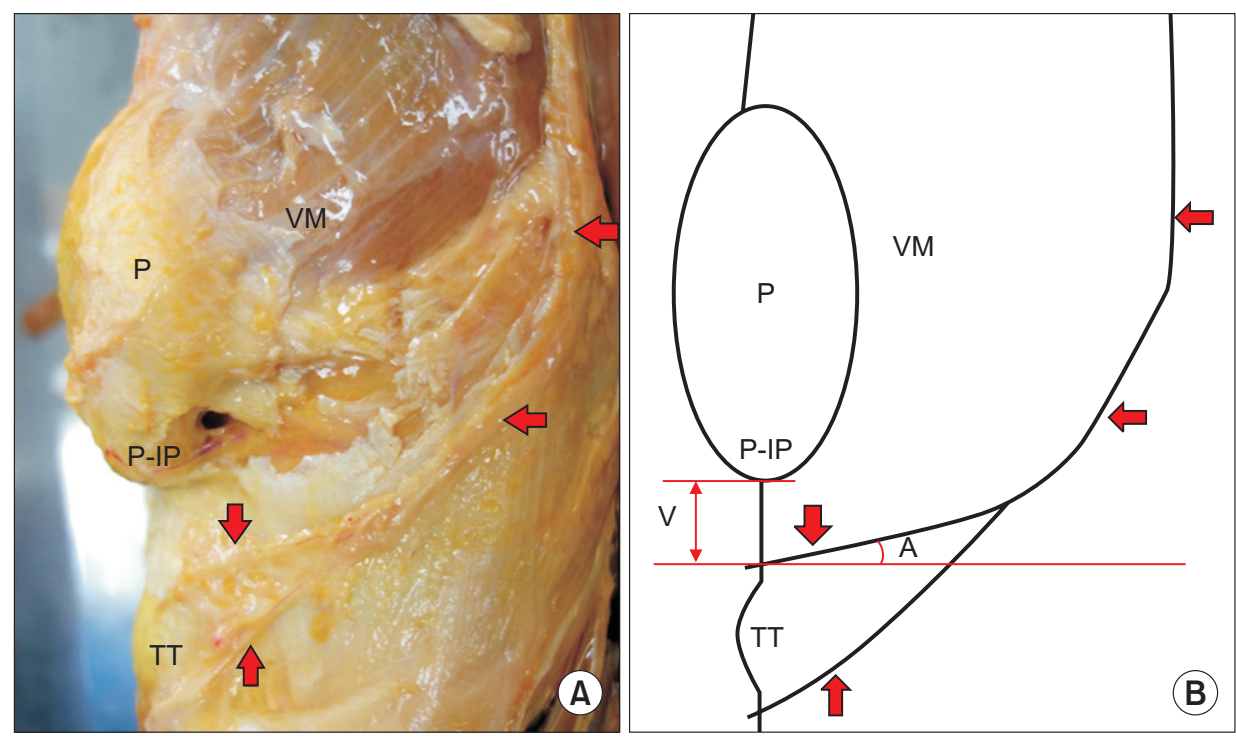

Fig. 2. (A) Anteromedial view of a dissected right knee showing the arc-like course and location of terminal branch of the IPBSN (arrow). (B) Schematic illustration demonstrating anatomical landmarks and relative location of the IPBSN (arrow). The vertical distance was from the inferior pole of the patella to the point where the terminal branch meets the reference line, connecting inferior pole of the patella and tibial tuberosity. The acute angle was the angle between terminal branch of the IPBSN and a perpendicular line to the reference line. VM, vastus medialis muscle; $\mathrm{P}$, patella; P-IP, inferior pole of patella; TT, tibial tuberosity; V, vertical distance; AA, acute angle; IPBSN, infrapatellar branch of saphenous nerve. 
terminal branch meets the reference line was measured. The acute angle of the direction of the path of the terminal branch of the IPBSN in relation to perpendicular line to the reference line was also measured (Fig. 2).

\section{Electrophysiologic study}

After an anatomical study of the positioning of the stimulation and recording site of IPBSN, nerve conduction studies were planned at the determined location to verify the clinical applicability of the location and to provide a reference value (Fig. 3). After approval of St. Paul's

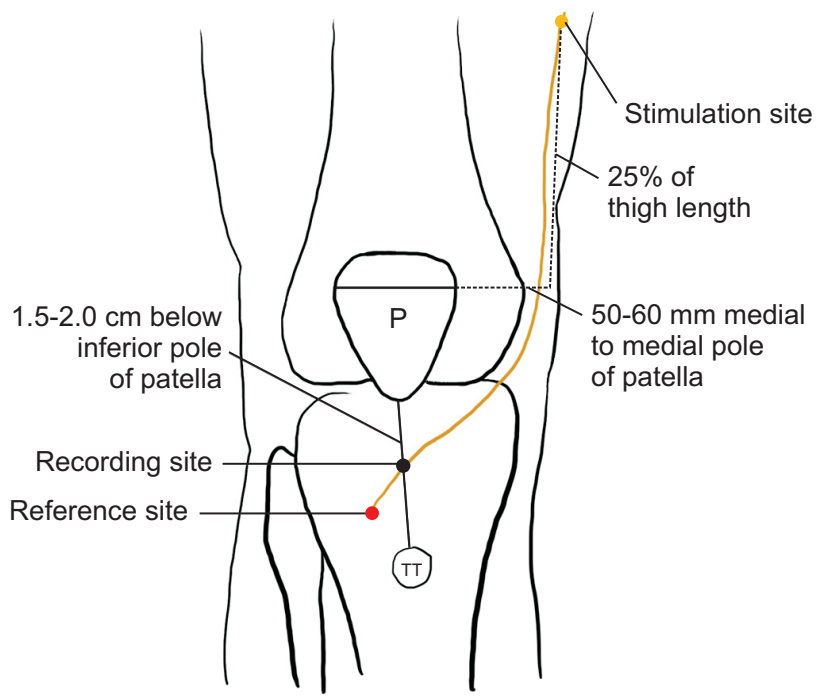

Fig. 3. Schematic illustration demonstrating nerve conduction study of right IPBSN. Stimulation was performed on the reference line on the $\mathrm{X}$-axis at $50-60 \mathrm{~mm}$ medial to the medial pole of patella, and at $25 \%$ of thigh length on the Y-axis. Recording electrode was attached 1.5-2.0 $\mathrm{cm}$ below the inferior pole of the patella. Reference electrode was placed $4 \mathrm{~cm}$ distal to the recording electrode in consideration of the direction of the IPBSN. P, patella; TT, tibial tuberosity; IPBSN, infrapatellar branch of saphenous nerve.
Hospital Institutional Review Board (No. PC16OISI0042) and written patient consent, we studied 37 healthy people (21 males and 16 females; median age, 30.95 years; range, 20-59 years) who had visited St. Paul's Hospital as volunteers who came in answer to posted clinical trial instruction, without any documented or anecdotal history of lower extremity lesions or other issues such as knee osteoarthritis or peripheral neuropathy. Those who presented with a history of surgical intervention were to one or both knees were also excluded. Right and left lower extremity nerve conduction studies were performed in all subjects, and total of 74 IPBSNs were studied. At the outset, all qualified subjects were required to have normal nerve conduction velocities on routine screening tests of the sural and superficial peroneal sensory nerves to exclude the possibility of peripheral neuropathy or polyneuropathy. Recording was made with subjects in a supine position with their legs extended. The recording was made with a sweep time of 10 minutes, stimulus duration of 0.1-0.2 minutes and stimulus intensity of 20-30 $\mathrm{mA}$, which was the maximum intensity without stimulation of the adjacent nerves. All electrodiagnostic studies were performed using a Sierra wave (Cadwell, Kennewick, WA, USA) electromyography. Skin temperature was maintained at $31^{\circ} \mathrm{C}-34^{\circ} \mathrm{C}$, and recording was planned to be performed at the optimal stimulation and recording site as measured by cadaveric study.

\section{RESULT}

\section{Location of optimal stimulation site}

The mean thigh length was $328.33 \pm 19.46 \mathrm{~mm}$ (range, $300-360 \mathrm{~mm}$ ). The mean value of $\mathrm{X}$-coordinate was $55.50 \pm 6.10 \mathrm{~mm}$. The mean value of Y-coordinate and the ratio of the distance to the thigh length were $84.58 \pm 22.46$ $\mathrm{mm}$ and $25.53 \% \pm 5.40 \%$, respectively (Table 1 ).

Table 1. The distance and relative location of the stimulation site of IPBSN

\begin{tabular}{ccccc}
\hline \multicolumn{2}{|c}{ Distance of stimulation site (mm) } & & Thigh length $^{\text {c) }}(\mathbf{m m})$ & $\begin{array}{c}\text { Relative vertical } \\
\text { location in thigh }^{\text {d) }}(\%)\end{array}$ \\
\hline X-coordinate & Y-coordinate & & & (\%) \\
\hline $55.50 \pm 6.10$ & $84.58 \pm 22.46$ & & $328.33 \pm 19.46$ & $25.53 \pm 5.40$ \\
\hline
\end{tabular}

Values are presented as mean \pm standard deviation.

IPBSN, infrapatellar branch of saphenous nerve.

${ }^{\text {a) }}$ From the medial pole of patella to the point where $\mathrm{X}$ - and Y-axis cross.

${ }^{\mathrm{b}}$ From the point where the nerve exiting the sartorius muscle to the point where $\mathrm{X}$ - and $\mathrm{Y}$-axis cross.

${ }^{\mathrm{c}}$ From the mid-point between anterior superior iliac spine (ASIS) and pubic tubercle to the medial pole of patella.

${ }^{d)}$ Ratio of Y-coordinate to the thigh length. 
The course of the IPBSN was classified into three types according to its relation to the sartorius muscle: anterior, penetrating, and posterior. The most common type was the penetrating type (58.3\%), followed by anterior type (33.3\%), and the posterior type (8.3\%) (Table 2).

\section{Location of recording site and branching pattern of terminal nerve of IPBSN}

The most common branching number of IPBSN was two $(75 \%)$, followed by single branch type $(25 \%)$. The location of terminal point of the nerve branches in relation to patella and tibial tuberosity was observed as organized in the Table 3. The location of terminal point of the nerve branches between the inferior pole of patella and tibial tuberosity was most commonly observed.

Table 2. Type of infrapatellar branches in relation to the sartorius muscle

\begin{tabular}{lccc}
\hline & $\begin{array}{c}\text { Total } \\
(\mathbf{n = 1 2})\end{array}$ & $\begin{array}{c}\text { Right } \\
(\mathbf{n = 6})\end{array}$ & $\begin{array}{c}\text { Left } \\
(\mathbf{n = 6})\end{array}$ \\
\hline Anterior type (\%) & 33.3 & 33.3 & 33.3 \\
\hline Penetrating type (\%) & 58.3 & 66.6 & 50.0 \\
\hline Posterior type (\%) & 8.3 & 0 & 16.6 \\
\hline
\end{tabular}

Table 3. Type of termination of branches in relation to the bony land marks: the patella and tibial tuberosity

\begin{tabular}{|c|c|c|c|}
\hline & \multirow{2}{*}{$\begin{array}{c}\text { Single } \\
\text { branch } \\
(n=3)\end{array}$} & \multicolumn{2}{|c|}{ Two branches $(\mathrm{n}=9)$} \\
\hline & & $\begin{array}{l}\text { Superior } \\
\text { branch }\end{array}$ & $\begin{array}{l}\text { Inferior } \\
\text { branch }\end{array}$ \\
\hline Over the patella & 0 & 2 & 0 \\
\hline $\begin{array}{l}\text { Between patella and } \\
\text { tibial tuberosity }\end{array}$ & 3 & 7 & 4 \\
\hline $\begin{array}{l}\text { Over or below tibial } \\
\text { tuberosity }\end{array}$ & 0 & 0 & 5 \\
\hline
\end{tabular}

Table 4. Location of recording site of IPBSN

\begin{tabular}{lr}
\hline & Value \\
\hline Vertical distance $^{\text {a) }}(\mathrm{mm})$ & $15.92 \pm 1.83(13-19)$ \\
Angle $^{\text {b) }}\left({ }^{\circ}\right)$ & $16.58 \pm 3.8(10-23)$ \\
\hline
\end{tabular}

Values are presented as mean \pm standard deviation (range).

IPBSN, infrapatellar branch of saphenous nerve.

${ }^{a}$ From the inferior pole of the patella to the point where the terminal branch meets the reference line.

${ }^{b)}$ Angle of the terminal branch of the IPBSN in relation to a perpendicular line to the reference line.
The mean distance from the inferior pole of patella to the terminal branch of IPBSN on the reference line was $15.92 \pm 1.83 \mathrm{~mm}$. The mean angle of the terminal branch of the IPBSN in relation to a perpendicular line to the reference line was $16.58^{\circ} \pm 3.68^{\circ}$ (Table 4 ).

\section{Reference values of sensory nerve conduction studies of IPBSN}

Among 37 adults, total of 74 IPBSN sensory nerve potentials were obtained (Fig. 4). Stimulation was performed on the reference line on the $\mathrm{X}$-axis at $50-60 \mathrm{~mm}$ medial to the medial pole of patella, and at $25 \%$ of thigh length (mean thigh length, $369.73 \pm 3.48 \mathrm{~mm}$; range, $300-440 \mathrm{~mm}$ ) on the Y-axis. A recording electrode was attached 1.5 to $2.0 \mathrm{~cm}$ below the inferior pole of the patella, and reference electrode was placed $4.0 \mathrm{~cm}$ distal to the recording electrode in consideration of the direction of the IPBSN. The mean peak latency of sensory nerve action potential (SNAP) was $4.11 \pm 0.30 \mathrm{~ms}$ and mean amplitude of SNAP was $4.16 \pm 1.49 \mu \mathrm{V}$, and there was no signifi-

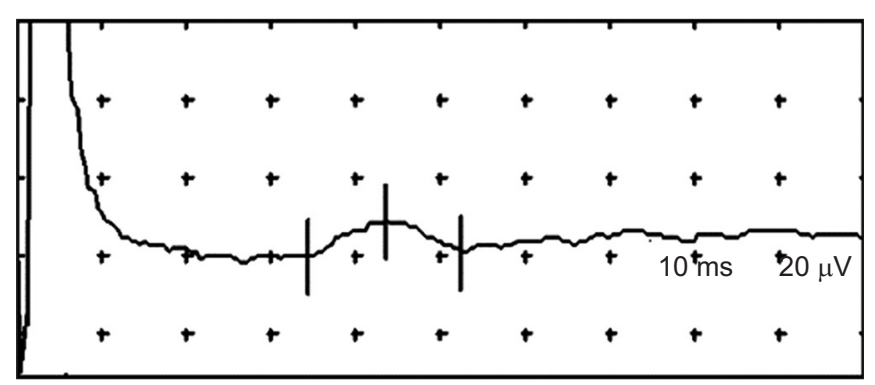

Fig. 4. Sensory nerve conduction study by obtained optimal stimulation and recording site for IPBSN (infrapatellar branch of saphenous nerve).

Table 5. Measurement of nerve conduction study of infrapatellar branch of saphenous nerve

\begin{tabular}{lcc}
\hline & $\begin{array}{c}\text { Peak latency } \\
(\mathbf{m s})\end{array}$ & $\begin{array}{c}\text { Amplitude } \\
(\boldsymbol{\mu V})\end{array}$ \\
\hline Male & $4.10 \pm 0.32$ & $4.30 \pm 1.67$ \\
Female & $4.13 \pm 0.28$ & $3.95 \pm 1.19$ \\
\hline Age (yr) & & \\
\hline $20-29$ & $4.07 \pm 0.26$ & $4.04 \pm 1.36$ \\
\hline $30-39$ & $4.21 \pm 0.34$ & $4.34 \pm 1.70$ \\
$40-49$ & $3.95 \pm 0.21$ & $3.60 \pm 1.27$ \\
\hline $50-59$ & $3.96 \pm 0.29$ & $4.05 \pm 0.40$ \\
\hline Total (reference values) & $4.11 \pm 0.30$ & $4.16 \pm 1.49$ \\
\hline
\end{tabular}

Values are presented as mean \pm standard deviation. 
cant correlation between the mean values of each group divided by age and gender (Table 5).

\section{DISCUSSION}

The goal of this study was to identify the optimal stimulation and recording point for use and reference value of IPBSN nerve conduction studies, through cadaveric and clinical study.

This study contributed to our understanding of the course of IPBSN. It exits the sartorius muscle to subcutaneous in thigh, extends distally toward knee and terminates at the level of the patella ligament.

Since IPBSN runs superficially right below the knee, there is significant chance of injury due to trauma or and iatrogenic procedure [7-10]. Clinically, patients with IPBSN lesion complain of pain and sensory disturbance such as numbness, lack of feeling, or paresthesia on the anteroinferior and medial knee. These clinical symptoms may masquerade as pes anserine bursitis, patellofemoral disorder, tear of medial meniscus and/or arthritis of knee. Also, IPBSN neuropathy is often misinterpreted as lumbar radiculopathy or femoral neuropathy. This is why proper electrophysiological evaluation of IPBSN is fundamental to the evaluation of sensory disturbance of knee.

There are number of studies about IPBSN conduction study. There is an electrophysiological study of IPBSN neuropathy regarding anterior cruciate ligament reconstruction with the hamstrings technique. In this study, authors applied electric stimulation $4 \mathrm{~cm}$ below the articular line, medial to the patella [12]. However, it is difficult to conduct such studies en bloc on IPBSN, due to its high variability. Also, although the recording site was not explicitly stated, the orthodromic sensory nerve conduction study recorded on the medial surface of the leg does not clearly suggest a lesion of IPBSN. Furthermore, depending on the thigh circumference, SNAP may not be observed, or might be discerned only at low amplitude.

Another electrophysiological study of IPBSN set the stimulation site $2 \mathrm{~cm}$ below the patella in medial knee, and the recording site at $2 \mathrm{~cm}$ lateral to the femoral artery in the inguinal region using needle electrode [3]. As an aside, this particular method could lead to pain and complication(s) such as hematoma due to needle insertion. Moreover, the long distance between stimulation site and recording site cause phase cancellation and tem- poral dispersion, which cause diminished SNAP amplitude.

Likewise, there are only a few IPBSN conduction study and each study demonstrate different locations of stimulation and recording sites. Accordingly, proper localization of stimulation and recording site using anatomical landmarks for IPBSN conduction study is necessary. This is the first study to propose an ideal stimulation site and recording sites of the IPBSN with objective coordinates based on anatomical landmarks for effective IPBSN nerve conduction study.

In this study, optimal stimulation site was defined as 'the location where the IPBSN exits the sartorius muscle, penetrates the fascia lata and becomes superficial. This point enables the stimulation to reach directly over the nerve. Placement of the stimulator at the optimal location assures the highest SNAP amplitude with the least stimulus intensity. Also, stimulating the nerve at this location provides supramaximal stimulation with little current which minimizes the pain of patients and reduces the technical errors such as depolarizing nearby adjacent nerves.

In antidromic study of the sensory nerve, the entire nerve including motor branch is depolarized frequently, inducing a volume-conducted motor potential. This potential is often misinterpreted as SNAP in pathologic condition where sensory potential is absent. However, in this study, the possibility of volume conduction could be excluded because IPBSN is pure sensory branch of femoral nerve.

We identified three anatomical variations of the IPBSN in relation to the sartorius muscle. In this study, most common type was the penetrating type $(50.0 \%)$, which was consistent to previous studies [2,4], but contradictory to one of the studies [1] where the most common was anterior type. Pes anserine type was not observed in this study, which was observed in one of the previous studies [4]. This differences between the cadaveric studies may be due to the difference in number of specimen(s) or ethnic variation(s).

There are several cadaveric studies about injury of terminal branch of IPBSN with the history of surgery on knee. In this cadaveric study, the IPBSN commonly gave its terminal branches in between patella and tibial tuberosity, as was demonstrated in previous cadaveric study(s) [1]. This location is $15.92 \mathrm{~mm}$ below inferior pole 
of patella in average and is consistent with a IPBSN nerve conduction study, where stimulation site is $2 \mathrm{~cm}$ below the patella in medial knee [3]. In addition, we measured the acute angle of the terminal branch of the IPBSN in relation to a perpendicular line to the imaginary line connecting patella and tibial tuberosity (considering the arclike course of the IPBSN). The mean angle was $16.58^{\circ}$, which was consistent with the findings revealed by a previous cadaveric study [5].

Based on our findings, we recommend the optimal stimulation site for IPBSN nerve conduction study as approximately $5.0-6.0 \mathrm{~cm}$ medial to medial pole of the patella and $25 \%$ of thigh length proximal to the reference line, which connects medial and lateral pole of patella. Also, we recommend the optimal recording sites to be approximately $1.5-2.0 \mathrm{~cm}$ below inferior pole of patella and place the pair of recording electrodes along the course of IPBSN, which is approximately $17^{\circ}$ to the horizontal line.

In sensory nerve conduction studies, if sufficient SNAP of IPBSN was not observed, we moved the stimulator slightly anterior or posterior to the sartorius muscle, and the recording electrodes repositioned slightly proximally or distally between patella and tibial tuberosity regarding anatomical variations. In this process, muscle twitching occurred easily by direct muscle stimulation. In this case, the stimulation position, duration, and intensity were changed to avoid volume conduction artifact. Also, in order to select a clear stimulation location, patients were asked whether the pins-and-needles sensation occurred at the medial knee site during stimulation.

There are few limitations in our study. We dissected total of 12 lower limbs which are, relatively speaking, a small sample size. Also, the mean age of specimens was 69 years old, which is quite elderly. In electrophysiologic study, there was no significant correlation between the mean values of each group divided by age and gender, which may be due to the relatively small sample size. In addition and in accordance with the anatomical variation(s) mentioned above, there also exists the possibility that other sensory nerves in the vicinity may be stimulated in the electrophysiologic study. However, we have confirmed through clinical studies that this location is applicable to healthy adults of various ages. Moreover and as best as we can determine, our study is the first to demonstrate the optimal stimulation site, recording site and its reference value for IPBSN nerve conduction study, therefore, this study can serve as a starting point for further studies using a lager age variation and larger sample size.

In conclusion, we have explored and evaluated the anatomical location of optimal stimulation site and recording site for more effective IPBSN conduction study. The optimal stimulation site was located $5.0-6.0 \mathrm{~cm}$ medial to medial pole of the patella and $25 \%$ of thigh length proximal to the $\mathrm{X}$-axis, and the optimal recording site was located 1.5-2.0 cm below inferior pole of patella. Mean peak latency of SNAP was $4.11 \pm 0.30 \mathrm{~ms}$ and mean amplitude of SNAP was $4.16 \pm 1.49 \mu \mathrm{V}$. The findings of this study may also prove useful for IPBSN conduction study.

\section{CONFLICT OF INTEREST}

No potential conflict of interest relevant to this article was reported.

\section{REFERENCES}

1. Kalthur SG, Sumalatha S, Nair N, Pandey AK, Sequeria S, Shobha L. Anatomic study of infrapatellar branch of saphenous nerve in male cadavers. Ir J Med Sci 2015;184:201-6.

2. Le Corroller T, Lagier A, Pirro N, Champsaur P. Anatomical study of the infrapatellar branch of the saphenous nerve using ultrasonography. Muscle Nerve 2011;44:50-4.

3. Bademkiran F, Obay B, Aydogdu I, Ertekin C. Sensory conduction study of the infrapatellar branch of the saphenous nerve. Muscle Nerve 2007;35:224-7.

4. Ackmann T, Von During M, Teske W, Ackermann O, Muller P, Von Schulze Pellengahr C. Anatomy of the infrapatellar branch in relation to skin incisions and as the basis to treat neuropathic pain by cryodenervation. Pain Physician 2014;17:E339-48.

5. Gali JC, Resina AF, Pedro G, Neto IA, Almagro MA, da Silva PA, et al. Importance of anatomically locating the infrapatellar branch of the saphenous nerve in reconstructing the anterior cruciate ligament using flexor tendons. Rev Bras Ortop 2014;49:625-9.

6. Standring S. Gray's anatomy: the anatomical basis of clinical practice. 41st ed. New York: Elsevier; 2016.

7. Mistry D, O'Meeghan C. Fate of the infrapatellar branch of the saphenous nerve post total knee arthro- 
plasty. ANZ J Surg 2005;75:822-4.

8. Swanson AJ. The incidence of prepatellar neuropathy following medial meniscectomy. Clin Orthop Relat Res 1983;181:151-3.

9. Mochida H, Kikuchi S. Injury to infrapatellar branch of saphenous nerve in arthroscopic knee surgery. Clin Orthop Relat Res 1995;320:88-94.

10. Ikpeme JO, Gray C. Traumatic prepatellar neuralgia. Injury 1995;26:225-9.
11. Saal JA, Dillingham MF, Gamburd RS, Fanton GS. The pseudoradicular syndrome. Lower extremity peripheral nerve entrapment masquerading as lumbar radiculopathy. Spine (Phila Pa 1976) 1988;13:926-30.

12. Figueroa D, Calvo R, Vaisman A, Campero M, Moraga C. Injury to the infrapatellar branch of the saphenous nerve in ACL reconstruction with the hamstrings technique: clinical and electrophysiological study. Knee 2008;15:360-3. 